\title{
Preface to the Special Issue on Ultrafine Grained Materials
}

\author{
Yuntian T. Zhu - Suveen N. Mathaudhu - Mathias Göken • \\ Terence G. Langdon • Terry C. Lowe - S. Lee Semiatin • \\ Nobuhiro Tsuji $\cdot$ Yong Hao Zhao
}

Received: 10 June 2010/ Accepted: 10 June 2010/Published online: 1 July 2010

(C) Springer Science+Business Media, LLC 2010

This is the third special issue of Journal of Materials Science on ultrafine-grained (UFG) materials. This issue contains 45 selected papers presented at the Sixth International Symposium on Ultrafine-Grained Materials (UFG VI) held in Seattle, WA, United States, on February 14-18, 2010, as part of the 2010 TMS Annual meeting. The UFG VI symposium received over 170 abstracts, making it the largest symposium at the TMS meeting. The papers collected in this issue were reviewed according to the strict reviewing policies of the journal and only those meeting the quality required for regular papers were accepted for publication.

UFG materials include both submicrocrystalline materials with grain sizes in the range of $100-1000 \mathrm{~nm}$ and nanocrystalline materials with grain sizes below $100 \mathrm{~nm}$. Such materials usually have superior mechanical and physical properties, including high strength, improved

Y. T. Zhu (ه)

Department of Materials Science and Engineering, North Carolina State University, Raleigh, NC 27695-7919, USA

e-mail: ytzhu@ncsu.edu

\section{S. N. Mathaudhu}

Materials and Manufacturing Sciences Division, U.S. Army

Research Laboratory, Aberdeen Proving Ground,

MD 21005-5069, USA

e-mail: suveen.mathaudhu@us.army.mil

\section{Göken}

Department of Materials Science and Engineering,

Institute I University Erlangen-Nürnberg, Martensstrasse 5, 91058 Erlangen, Germany

e-mail: goeken@ww.uni-erlangen.de

\section{T. G. Langdon}

Departments of Aerospace \& Mechanical Engineering and Materials Science, University of Southern California, Los Angeles, CA 90089-1453, USA

e-mail: langdon@usc.edu corrosion resistance, higher wear resistance, etc. Two complementary approaches have been developed for synthesizing UFG materials. The first is the "bottom-up" approach in which UFG materials are assembled from individual atoms or from nanoscale building blocks such as nanoparticles. The second is the "top-down" approach in which existing coarse-grained materials are processed to produce ultrafine grains. The most successful top-down approaches involve the application of severe plastic deformation, in which materials are subjected to the imposition of strains that are typically larger than 4-6. Examples of severe plastic deformation include equal channel angular pressing (ECAP), high-pressure torsion (HPT), accumulative roll bonding (ARB), and other variants developed more recently. Most of the papers collected in this special issue are concerned with the top-down

T. C. Lowe

Manhattan Scientifics Incorporated, The Chysler Building 32nd Floor, 405 Lexington Avenue, New York, NY 10174, USA

e-mail: terry@mhtx.com

S. L. Semiatin

Air Force Research Laboratory, Materials \& Manufacturing

Directorate, WPAFB, OH 45433, USA

e-mail: Lee.Semiatin@wpafb.af.mil

N. Tsuji

Department of Materials Science and Engineering,

Kyoto University, Kyoto 606-8501, Japan

e-mail: nobuhiro.tsuji@ky5.ecs.kyoto-u.ac.jp

Y. H. Zhao

Department of Chemical Engineering and Materials Science, University of California, Davis, Davis, CA 95616-5294, USA e-mail:yhzhao@ucdavis.edu 
approach, while studies on other UFG-related topics are also included.

The area of UFG materials remains one of the hot topics in the field of materials science and engineering, and the UFG community is continuing to grow as evidenced by the increasing number of participants in UFG-related international conferences and symposia (More information on past and future conferences in this area can be found at http://www.nanoSPD.org/). Much progress on the investigation of important but difficult issues has been made recently, including in fracture toughness, fatigue, high thermal stability, and higher strength and ductility. This special issue has collected papers reporting on most of these new developments as well as on new processing methods, microstructures, properties, and modeling.

In the last few years, we have also seen more commercialization effort in the area of UFG materials. Continuous SPD processing techniques that are conducive to large-scale, low cost production in industrial facilities have been reported. However, the scientific community still faces some important issues, including improving fatigue strength, ductility, strength, and thermal stability. Other important topics to be studied include the irradiation resistance of UFG materials which are believed to be potential good candidates for building the next generation of nuclear power plants. In addition, crystalline defects and their role in the deformation behavior of bcc and hcp UFG metals and alloys have not been well studied. Previous studies reported so far have largely focused on fcc metals and alloys. A survey of the literature also reveals that investigations in the past have used mostly commercial alloys. These alloys have been designed for coarse-grained materials, and they may not be able to fully utilize the unique deformation mechanisms in UFG materials for the best mechanical properties. Therefore, the designing of new UFG alloys is needed to obtain the desired mechani$\mathrm{cal}$, thermal, and physical properties that will be required in order to overcome many limitations of current commercial alloys. It is our expectation that more studies and much progress will be made in these areas. 